Ciencia y Salud, Vol. III, No. 3, septiembre-diciembre, 2019 • ISSN (impreso): 2613-8816•ISSN (en línea): 2613-8824

DOI: https://doi.org/10.22206/cysa.2019.v3i3.pp7-13

\title{
PROTOTIPO DE VIDEO FRENZEL-VIDEO HEAD IMPULSE TEST DE BAJO COSTE
}

\section{Prototype of video Frenzel-Video Head impulse test low cost}

\author{
Frank Alberto Betances Reinoso ${ }^{\mathrm{a}}$, Tania Lopez Montes ${ }^{\mathrm{b}}$, Víctor Rodríguez Ontiveros ${ }^{\mathrm{c}} \mathrm{y}$ \\ Nicole de León ${ }^{d}$
}

Recibido: 16 de febrero, 2019 • Aprobado: 21 de abril, 2019

Cómo citar: Betances Reinoso FA, Lopez Montes T, Rodríguez Ontiveros VM, de León N. Prototipo de video Frenzel-Video Head impulse test de bajo coste. cysa [Internet]. 25 de noviembre de 2019 [citado 25 de noviembre de 2019];3(3):7-13. Disponible en: https://revistas.intec.edu.do/index.php/cisa/article/view/1545

\section{Resumen}

Antecedentes: desde la introducción de las gafas de Frenzel se han desarrollados múltiples sistemas que permiten no permiten no solo observar el nistagmo, sino documentarlo y valorar su evolución y respuesta al tratamiento; pero el costo pero el costo de dichos equipos limita su uso en los países en vías de desarrollo.

Objetivo: desarrollar un prototipo de Video Frenzel / Video Head impulse test de bajo coste.

Materiales: utilizamos una cámara de video USB con visión nocturna, un acelerómetro/giroscopio USB, una impresora 3Dstandard, unas gafas de realidad virtual para móviles y el software $\mathrm{MATLAB}^{\circ}$ para desarrollar el programa que registra los movimientos oculares y cefálicos. Comparamos el prototipo frente a las gafas de Frenzel mediante la valoración de una serie de pacientes.

Resultados: en total incluimos 10 pacientes en nuestro estudio, con una media de edad de 62 años, el prototipo

a Servicio de Otorrinolaringología, Hospital Universitario Donostia, San Sebastián, Gipuzkoa, España. Correo-e: drbetances@hotmail.com

b Servicio de Oftalmología - Royal Cornwall Hospital, Truro, Cornwall, Reino Unido. Correo-e: tania1_21lm@hotmail.com

\section{Abstract}

Background: Since the introduction of frenzel glasses, multiple systems have been developed that allow us not only to observe the nystagmus, but also to document it, allowing us to assess its evolution and response to treatment, but the cost of such equipment limits its use in developing countries.

Objective: Develop Video Frenzel / Video Head impulse low cost test.

Materials: We use a USB video camera with night vision, a USB accelerometer / gyroscope, a standard $3 \mathrm{~d}$ printer, a virtual reality glasses for mobile phones and the MATLAB ${ }^{\circ}$ software to develop the program that records eye movements and cephalic movements. We compared the prototype to the frenzel glasses by assessing a series of patients.

Results: In total we included 10 patients in our study, with an average age of 62 years, the prototype allowed us to record vestibular maneuvers and assess their effectiveness.

\footnotetext{
${ }^{\mathrm{c}}$ Escuela Universitaria Politécnica de Teruel, Teruel, Teruel, España Correo-e: victor_rod_ont@hotmail.com

d Servicio Medicina Interna - Columbia Presbyterian Hospital, New York, Estados Unidos. Correo-e: dra.deleon33@gmail.com
} 
nos permitió registrar las maniobras vestibulares y valorar su eficacia.

Conclusión: hemos desarrollado un dispositivo de bajo coste con unas características similares a los equipos comerciales tanto en la función, peso, comodidad para el paciente y facilidad de uso para el médico.

Palabras clave: video-Frenzel; V-HIT; nistagmo; vértigo; vestibular.

\section{Introducción}

La patología relacionada con el vértigo es muy habitual siendo una de las causas más frecuentes de consulta en atención primaria y de otorrinolaringología; puede ocurrir a cualquier edad, aunque predomina entre los 40-60 años, y es más frecuente en el sexo femenino ${ }^{1}$.

Para el diagnóstico del vértigo es necesario realizar una buena anamnesis y contar con el material adecuado para completar la exploración, sobre todo que tenga la capacidad para documentar los movimientos oculares característicos del vértigo de origen periférico.

Las gafas Frenzel son una herramienta extremadamente útil para el correcto diagnóstico de pacientes con vértigo. Básicamente, consisten en una serie de lentes de 15-20 dioptrías que permiten anular la fijación visual. Poseen un sistema de iluminación lateral que facilita la visualización del nistagmo en cualquier posición de la mirada, pero tienen el inconveniente que no anulan completamente la visión.

Los sistemas que incorporan grabación de video, aunque más costosos, son siempre preferibles a las gafas de Frenzel, ya que permiten el registro y la visualización del examen por el paciente o familiares. otra ventaja de las video-Frenzel es que anulan completamente la fijación visual ${ }^{2}$.

8 | Ciencia y Salud 2019; 3(3): 7-13 • Artículo original
Conclusion: We have developed a low cost device with characteristics similar to commercial equipment in terms of function, weight, comfort for the patient and ease of use for the physician.

Keywords: Video-Frenzel; V-HIT; nystagmus; dizziness; vestibular.

La prueba de impulso cefálico (head impulse test) consiste en una herramienta sencilla y rápida para evaluar la función del reflejo vestíbulo ocular. la implementación de una cámara de alta velocidad y un acelerómetro permiten el registro de dicha prueba (Video head impulse test / V-HIT) ${ }^{3}$.

El alto costo de estos equipos limita su disponibilidad en los países en vías de desarrollo; la falta de dichos equipos se traduce en diagnósticos incorrectos y atenciones médicas de baja calidad, por lo que decidimos desarrollar un prototipo de bajo coste de un video Frenzel-Video head impulse test que permita a centros hospitalarios, con un presupuesto limitado, adquirir dichos equipos.

\section{Materiales y métodos}

Comparamos el prototipo con las gafas de Frenzel clásicas durante la valoración de una serie de pacientes derivados desde atención primaria a consultas externas de ORL del Hospital General de Teruel, para descartar la posibilidad de vértigo posicional. Todos los pacientes fueron valorados por el mismo otorrinolaringólogo, a todos se le realizó la maniobra Dix-Hallpike primero con gafas de Frenzel, 10 minutos después se repitió la maniobra con el prototipo.

En total se incluyeron 10 pacientes; todos firmaron un consentimiento informado, donde autorizaron su inclusión en el estudio. 
Gafas de realidad virtual móvil: realmente son carcasas que no tienen pantalla propia ni procesador, sino que están preparadas para albergar un teléfono móvil, en el cual se reproducirán las imágenes. En nuestro caso, utilizamos un modelo de bajo coste con un precio aproximado de 10 euros.

Cámara de alta definición con leds infrarrojos: se trata de un módulo USB de cámara de alta velocidad con una definición de 1080 P, capaz de grabar 120 FPS, equipada con un sensor CMOS OV2710 que permite grabar imágenes nítidas. Diez leds infrarrojos permiten captar los movimientos oculares en completa oscuridad y sin fijación visual, compatible con Windows y MAC, la cámara tuvo un coste de 35 euros a través de Aliexpress.

Acelerómetro y giroscopio: utilizamos un sensor MPU-9250 que combina dos chips: el MPU-6500, el cual contiene un giroscopio y un acelerómetro, ambos de 3 ejes, y el AK8963, que cuenta con un magnetómetro de 3 ejes. El MPU-9250 es un dispositivo pequeño, lo que lo hace ideal para incorporarlo en la mayoría de los proyectos que requieran la medición de la orientación o posicionamiento. Este sensor utiliza el protocolo de comunicación I2C, que lo hace compatible con cualquier microcontrolador que incluya este bus de comunicación.

Para hacer uso del MPU-9250 en este trabajo, se utiliza un microcontrolador ARM cortex-M3 STM32F103C8T6, que incorpora varios puertos I2C y un puerto USB, por el cual se envían los datos al ordenador emulando un puerto serie. Este microcontrolador puede ser alimentado vía USB, permitiendo una integración perfecta dentro de la carcasa. El precio del microcontrolador en Amazon es de aproximadamente 8 euros, mientras que del MPU-9250 es de 4 euros.

Impresora 3D: utilizamos una impresora 3D modelo Prusa i3, tiene una resolución máxima de 60 micras para imprimir la carcasa de la cámara que permite enfocar el globo ocular. La misma fue facilitada por la Escuela Universitaria Politécnica de Teruel.

Programa informático para registro de los movimientos oculares y de la cabeza: el programa informático fue desarrollado por la Escuela Universitaria Politécnica de Teruel. Registra los movimientos sacádicos del ojo mediante el seguimiento de la pupila y los datos aportados por el sensor del acelerómetro/ giroscopio con respecto a la cabeza. El programa fue creado mediante $M^{2} T_{L A B}{ }^{\circ}$, el cual es una herramienta de software matemático que ofrece un entorno de desarrollo integrado con un lenguaje de programación propio (lenguaje $\mathrm{M})^{4}$.

\section{Resultados}

Hemos utilizado el prototipo en la consulta de otorrinolaringología del Hospital General de Teruel como prueba complementaria durante la exploración vestibular de 10 pacientes (tabla 1 ) derivados por sospecha de vértigo posicional, la media de edad de los pacientes fue de 62 ańos con un rango de 55 a 73 años, el $70 \%$ fue de sexo femenino.Utilizamos el prototipo para registrar la maniobra de Dix-Hallpike durante la exploración clínica, de los 10 pacientes derivados por sospecha de vértigo de origen periférico la maniobra fue positiva con 6 pacientes, de estos, 4 tenían afectado el canal semicircular posterior derecho, un paciente el canal semicircular posterior y, por último, una paciente el canal semicircular horizontal izquierdo. Documentar la exploración permitió compartirla con los compańeros del servicio, realizar un diagnóstico de certeza y controlar la evolución. Dentro de las valoraciones hechas, sin lugar a dudas, la mayor utilidad del prototipo fue para controlar la evolución de la paciente con una canalolitiasis clínica de canal semicircular horizontal izquierdo. El prototipo permitió compartir y documentar tanto la exploración como la evolución, tras realizar las maniobras de reposición. 
Tabla 1.Serie de casos incluidos en el estudio

\begin{tabular}{|c|c|c|c|}
\hline Edad & Sexo & $\begin{array}{c}\text { Dixhallpike } \\
\text { Gafas de Frenzel }\end{array}$ & $\begin{array}{c}\text { Dixhallpike } \\
\text { Prototipo } \\
\text { VHIT }\end{array}$ \\
\hline 55 años & Femenino & Normal & Normal \\
\hline 73 años & Femenino & CSH Izquierdo & $\begin{array}{c}\text { CSH } \\
\text { Izquierdo }\end{array}$ \\
\hline 65 años & Masculino & CSP Derecho & CSP Derecho \\
\hline 67 años & Femenino & CSP Derecho & CSP Derecho \\
\hline 59 años & Femenino & Normal & Normal \\
\hline 58 ańos & Femenino & Normal & Normal \\
\hline 63 años & Masculino & CSP Izquierdo & CSP Izquierdo \\
\hline 63 años & Femenino & CSP Derecho & CSP Derecho \\
\hline 57 años & Masculino & CSP Derecho & CSP Derecho \\
\hline 60 años & Femenino & Normal & Normal \\
\hline
\end{tabular}

Fuente: elaboración propia.

El prototipo (imagen 1) permite grabar y reproducir en tiempo real en la pantalla del ordenador los movimientos oculares, sin necesidad de tener instalado un programa informático. La cámara es detectada sin problemas tanto en ordenadores con Windows como en MacOS.

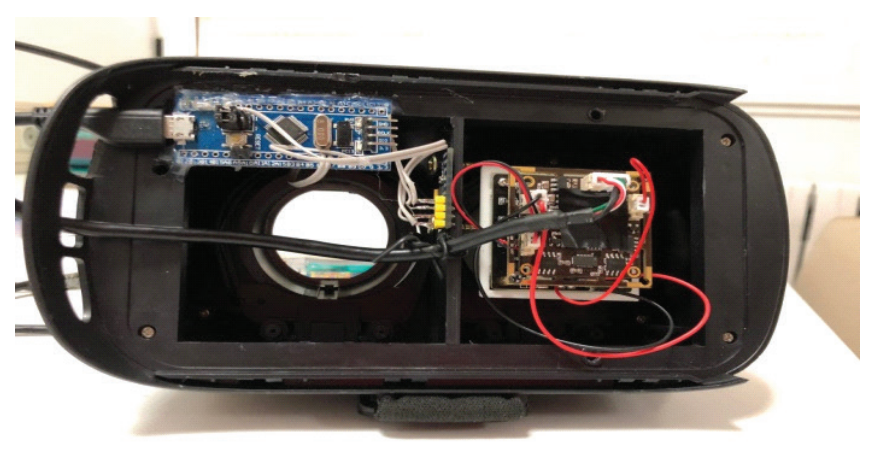

Imagen 1. Prototipo Videofrenzel.

Fuente: elaboración propia

La carcasa cuenta con una rendija que al cerrarla elimina la fijación visual, por lo que es un excelente instrumento para capturar los resultados de una maniobra de Dix-Hallpike (imagen 2), por otro lado, al abrir la rendija, el paciente pueda fijar la mirada, permitiendo realizar un head impulse test.

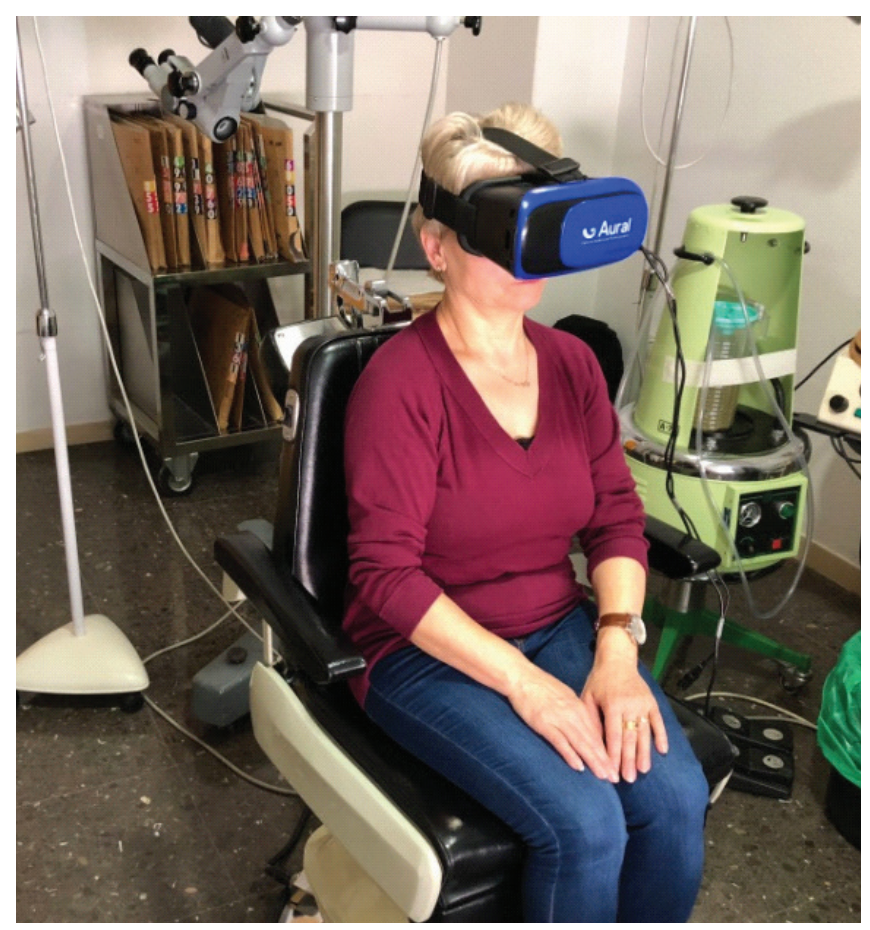

Imagen 2. Paciente sentada en silla de exploración previo a realización de maniobras de Dix-Hallpike.

Fuente: elaboración propia

La cámara de alta velocidad es capaz de detectar los movimientos sacádicos y el sensor de movimiento MPU-9250 permite corroborar si existe algún déficit vestibular, gracias al programa informático desarrollado en MATLAB $^{\circledast}$ (imágenes 3, 4, 5).

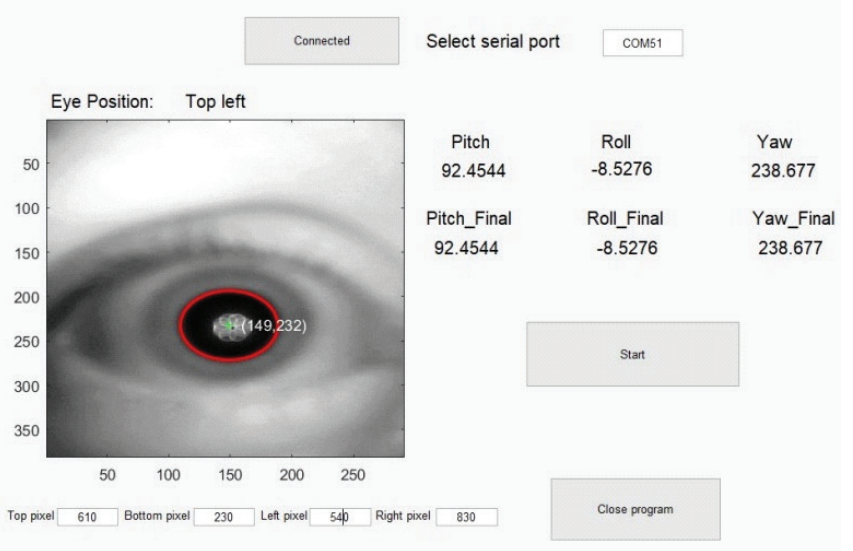

Imagen 3. Captura de imagen del ojo en posición central.

Fuente: elaboración propia 


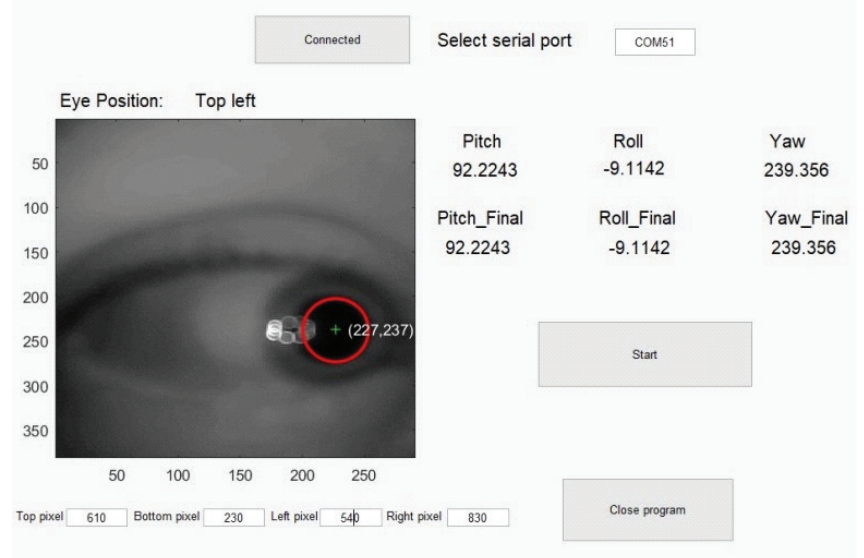

Imagen 4. Captura de imagen del ojo mirando hacia dentro.

Fuente: elaboración propia

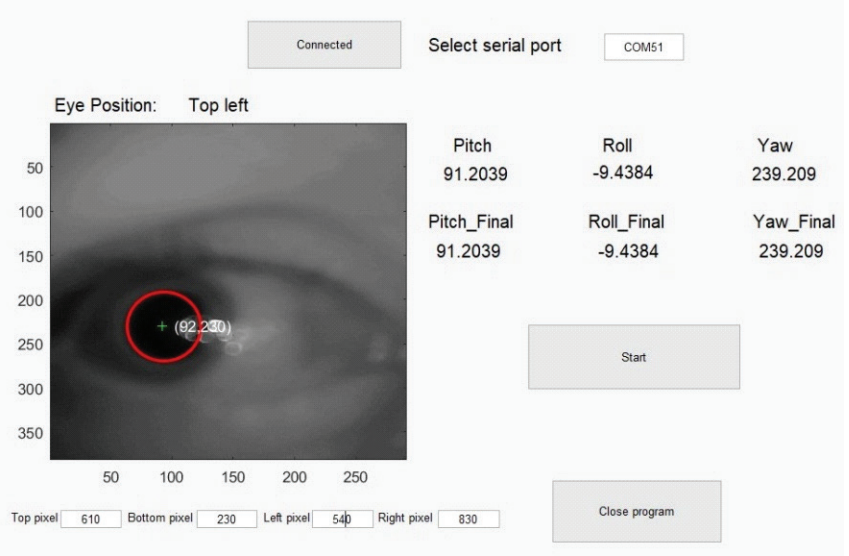

Imagen 5. Captura de imagen del ojo mirando hacia afuera. Fuente: elaboración propia

\section{Discusión}

Los trastornos del equilibrio, entre ellos el vértigo periférico, representan una de las causas más frecuentes de asistencia a la consulta médica y, en muchos casos, su diagnóstico genera muchas dudas, sobre todo por lo heterogéneo de su presentación ${ }^{5-10}$, ya que el vértigo puede producirse por diferentes procesos, entre ellos: enfermedades víricas, vasculares, desmielinizantes, tumores, etc ${ }^{5-10}$.
El vértigo posicional paroxístico benigno constituye la forma más frecuente de vértigo periférico, con predominio en las mujeres ${ }^{1}$. El diagnóstico es esencialmente clínico mediante la realización de las maniobras de Dix-Hallpike ${ }^{11}$, en ellas se evalúa la aparición de nistagmo al pasar el paciente desde la posición de sentado a decúbito supino con la cabeza girada $45^{\circ}$ de uno a otro lado. Durante la realización es importante el uso de las gafas de Frenzel para evitar la fijación visual. Un problema de las gafas clásicas es que no evitan completamente la fijación; nuestro prototipo, al sellar la región ocular, impide completamente la fijación visual, permitiendo valorar mucho mejor el nistagmo de origen periférico.

En el año 2004 Guevara et al., ${ }^{12}$ publicaron un artículo donde explican cómo fabricar un video Frenzel de bajo coste, el mismo está basado en una cámara web adaptada a unas gafas de seguridad. En nuestro prototipo utilizamos una cámara de alta definición con infrarrojos, con lo cual obtenemos imágenes más nítidas. $\mathrm{Al}$ mismo tiempo, fabricamos una carcasa que nos asegura que no penetre la luz, impidiendo la fijación visual completamente.

Park et al., en el año $2017^{13}$ desarrolló un video oculográfico de bajo coste. Es un aparato que es capaz de detectar los movimientos sacádicos. A diferencia de nuestro prototipo, para detectar los movimientos oculares utilizan un mini ordenador rapsberry Pi integrado a la carcasa. En nuestro caso, es utilizado un programa informático basado en MATLAB ${ }^{\circledR}$.

La prueba de impulso cefálico es una prueba simple, sencilla y rápida, ideada originalmente dentro del examen físico otoneurológico básico en la entrevista inicial del paciente ${ }^{14}$.

Esta prueba (que puede ser encontrada en la literatura como head impulse testo head thrust test) está basada en el reflejo vestíbulo-ocular. Consiste en la 
compensación de movimientos cefálicos por parte de los músculos extrínsecos oculares, de tal forma que la mirada pueda permanecer fija en un objeto pese a los movimientos de la cabeza, por bruscos y rápidos que estos sean ${ }^{14}$.

El diagnóstico de los pacientes con trastornos del equilibrio no solo debe incluir el historial clínico y la revisión física, sino también pruebas objetivas y cuantitativas ${ }^{15}$. Pérez-Férnandez et al., ${ }^{16}$ realizaron un trabajo donde afirman que el estudio del reflejo vestíbulo-ocular con un sistema de registro video asistido (vHIT, GN Otometrics) es un procedimiento que aporta hallazgos diferentes a los que se obtienen en el estudio clínico convencional. En el contexto de la consulta especializada aporta información adicional que permite mejorar la precisión diagnóstica del estudio a la cabecera del paciente.

El costo aproximado de unas gafas de Frenzel es superior a 400 euros, el costo de un videonistagmógrafo o un V-HIT es superior a los 10,000 euros. En nuestro caso, invertimos menos de 50 euros en el desarrollo del equipo, por lo que creemos que es una opción a tener en cuenta para complementar la consulta de trastornos vestibulares.

\section{Conclusión}

El videonigstamógrafo y el video head impulse test (V-HIT) son herramientas muy importantes para el adecuado diagnóstico y seguimiento de los trastornos vestibulares. El alto costo de dichos equipos limita la generalización de los mismos, por lo que consideramos que un dispositivo con características similares y con un coste significativamente menor ofrecería, a la mayoría de los servicios de consultas, la adquisición de un instrumento que permita valorar los trastornos vestibulares y registrar de manera objetiva las diversas maniobras, tanto diagnósticas como terapéuticas. Debido a su bajo coste, también podría ser una herramienta al alcance del médico de urgencias, para el adecuado cribado de los mareos.

\section{Bibliografía}

1. Gil-Carcedo LM, Vallejo LA, Gil-Carcedo E. Otología: Síndrome vestibulares periféricos. (3rd ed.). Madrid: Editorial Panamericana; 2011.

2. Hain T. Frenzel goggles. Dizziness-And-balance. com. Chicago [Internet]. [Consultado18 de marzo 2018]. Available from: http://www.tchain. com/otoneurology/practice/frenzels.htm.

3. Breinbauer H, Anabalón JL, Aracena K, Nazal D, Baeza $\mathrm{M}$ de los Á. Experiencia en el uso video-Impulso Cefálico (vHIT) en la evaluación del reflejo vestíbulo-ocular para el canal semicircular horizontal. Revista de otorrinolaringología y cirugía de cabeza y cuello. 2013;73(2):115-24.

4. MathWorks. MATLAB. Massachusetts [Internet]. [Consultado 18demarzo 2018]. Availablefrom:https://es.mathworks.com/products/new_products/ latest_features.html?s_tid=hp_spot_r2013a_0213.

5. Brand T, Dietrich M. Pathological eye-head coordination in roll: tonic ocular tilt reaction in mesencephalic and medullary lesions. Brain 1987;110: 649-66.

6. Brandt $T$. Vertigo. Its multisensory syndromes. London: Springer Ver-lag; 1991.

7. Buttner U, Brandt T. Clinical neurology. Ocular motor disorders of therotatory nystagmus arising from the semicircular canal via the singularnerve. Sem Hearing 1989;10: 191-5.

8. Linthicum FH Jr, Waldorf R, Luxford WM, Caltogirone S. Infrared/video ENG recording of eye movements to evaluate the inferior vestibular nerve using the minimal caloric test. Otolaryngol Head Neck Surg 1988;98: 207-10.

9. Wipperman J. Dizziness and vertigo. Prim Care Clin Office Pract. 2014;41:115-31.

10. Hanley, K O'Dowd T. Symptoms of vertigo in general practice: a prospective study of diagnosis. Br J Gen Pract. 2002;52:809-12. 
11. Dix R, Hallpike CS. The pathology, symptomatology and diagnosis of certain common disorders of the vestibular system. Ann OtolRhinolLaryngol 1952;6:765-78.

12. Guevarra JR, Chiong CM. Low cost frenzel goggles. Philippine J Otol HNS. 2004;19:1-2.

13. Park J, Kong Y, Nam Y. A low-cost video-oculography system for vestibular function testing. 2017; p. 4078-81.

14. Breinbauer K HA, Anabalón B JL. Prueba de impulso cefálico. Revista de otorrinolaringología y cirugía de cabeza y cuello. Agosto de 2011;71(2):123-30.
15. Said-MartínezJ, Izita-Rosales A. Nuevas técnicas en el diagnóstico de pacientes con vértigo. An Orl mex 2011;56(1):1-10.

16. Pérez-Fernández N, Gallegos-Constantino V, Barona-Lleo L, Manrique-Huarte R. Exploración clínica y videoasistida del reflejo vestíbulo-oculomotor: análisis comparativo. Acta Otorrinolaringológica Española. Noviembre de 2012;63(6):429-35. 